\title{
Bioburden after Staphylococcus aureus Inoculation in Type 1 Diabetic Rats Undergoing Internal Fixation
}

Nga L. Brown, M.S. Michael B. Rose, M.D. Gert Blueschke, M.D. Eugenia H. Cho, B.S. Mark H. Schoenfisch, Ph.D. Detlev Erdmann, M.D., Ph.D., M.H.Sc. Bruce Klitzman, Ph.D. Durham and Chapel Hill, N.C.
Summary: Fracture stabilization in the diabetic patient is associated with higher complication rates, particularly infection and impaired wound healing, which can lead to major tissue damage, osteomyelitis, and higher amputation rates. With an increasing prevalence of diabetes and an aging population, the risks of infection of internal fixation devices are expected to grow. Although numerous retrospective clinical studies have identified a relationship between diabetes and infection, currently there are few animal models that have been used to investigate postoperative surgical-site infections associated with internal fixator implantation and diabetes. The authors therefore refined the protocol for inducing hyperglycemia and compared the bacterial burden in controls to pharmacologically induced type 1 diabetic rats after undergoing internal fracture plate fixation and Staphylococcus aureus surgical-site inoculation. Using an initial series of streptozotocin doses, followed by optional additional doses to reach a target blood glucose range of 300 to $600 \mathrm{mg} / \mathrm{dl}$, the authors reliably induced diabetes in 100 percent of the rats $(n=16)$, in which a narrow hyperglycemic range was maintained 14 days after onset of diabetes (mean \pm SEM, $466 \pm$ $16 \mathrm{mg} / \mathrm{dl}$; coefficient of variation, 0.15 ). With respect to their primary endpoint, the authors quantified a significantly higher infectious burden in inoculated diabetic animals (median, $3.2 \times 10^{10}$ colony-forming units $/ \mathrm{mg}$ dry tissue) compared with inoculated nondiabetic animals $\left(7.2 \times 10^{4}\right.$ colony-forming units/ $\mathrm{mg}$ dry tissue). These data support the authors' hypothesis that uncontrolled diabetes adversely affects the immune system's ability to clear Staphylococcus aureus associated with internal hardware. (Plast. Reconstr. Surg. 134: 00, 2014.) iabetes mellitus affects 26 million individuals in the United States, ${ }^{1}$ causing a growing incidence of nonhealing ulcers with limb and digit amputations. Diabetic patients are at increased risk of bone fractures, often requiring internal hardware..$^{2-4}$

Over 2 million orthopedic procedures are performed annually involving implantable materials. ${ }^{5}$ Indwelling devices are associated with a variety of complications, the most important of which is infection causing soft-tissue damage and osteomyelitis. ${ }^{4,6,7}$ These infections are generally difficult

From Kenan Plastic Surgery Research Laboratories, Duke University Medical Center; the Department of Biomedical Engineering, Duke University; and the Department of Chemistry, University of North Carolina.

Received for publication October 10, 2013; accepted February 6, 2014.

The first two authors contributed equally to this work.

Copyright (C) 2014 by the American Society of Plastic Surgeons

DOI: 10.1097/PRS.0000000000000434 to manage and necessitate prolonged antibiotic therapy and revision surgery. ${ }^{5,8,9}$ The overall infection rate for implanted hardware is approximately 5 percent, with 40 percent of these infections caused by Staphylococcus aureus. ${ }^{6}$

Diabetic patients undergoing orthopedic surgery have increased rates of perioperative complications. ${ }^{7,10}$ Several large-scale case series studied the predictability of postoperative infection, ${ }^{11-15}$ with infection rates in diabetic patients of 7 to 10 percent compared with 1 percent in nondiabetics. Currently, there are few experimental models with which to study postoperative surgical-site infections in diabetic animals after hardware implantation, limiting quantitative evaluation of new therapies. Therefore, we investigated an experimental model for type 1 diabetes in rats

Disclosure: None of the authors has a financial interest in any of the products or devices mentioned in this article. 
Plastic and Reconstructive Surgery • October 2014

that allows quantification of the bacterial burden after hardware implantation and inoculation with S. aureus.

\section{MATERIALS AND METHODS}

\section{Fixation Plates}

Stainless steel fracture plates $(2.0 \mathrm{~mm}$; Synthes, Paoli, Pa.) were cut into 1-cm segments. Selftapping stainless steel cortical screws were cut to a length of 4 to $5 \mathrm{~mm}$.

\section{Bacterial Husbandry}

A methicillin-sensitive strain of $S$. aureus derived from a previously resistant strain of Colindale (termed COL, thought to be strain 9204) was provided by Dr. Vance Fowler, Section of Infectious Diseases, Duke University. Stationary phase cultures of $S$. aureus were grown from $-80^{\circ} \mathrm{C}$ stock overnight at $37^{\circ} \mathrm{C}$ in an 8-ml tryptic soy broth tube. Aliquots of $100 \mu \mathrm{l}$ were transferred into fresh $8-\mathrm{ml}$ tryptic soy broth tubes and incubated at $37^{\circ} \mathrm{C}$ for 5 hours to obtain a log phase culture. This yielded $2 \times 10^{8}$ colony-forming units $/ \mathrm{ml}$ as estimated by comparison to the $1.0 \mathrm{McF}$ arland standard. Serial dilution and culture plating were used to confirm reproducibility.

\section{In Vivo Studies}

This protocol was approved by the Duke University Animal Care and Use Committee. Male CD Rats (150 to $200 \mathrm{~g}$ ) were obtained from Charles River Laboratories (Raleigh, N.C.). Rats were given streptozotocin (VWR International, Radnor, Pa.) injections of $40 \mathrm{mg} / \mathrm{kg}$ in citrate buffer consecutively for 3 days, with a fasting period of 8 hours on day 1 before injection. ${ }^{3,9}$ Rats were given water supplemented with $15 \mathrm{~g} /$ liter sucrose for 48 hours to protect from streptozotocin-induced insulin release. Forty-eight hours after the third injection, 3-hour fasting blood glucose measurements were taken through the tail vein using a standard glucometer (One Touch Ultra; LifeScan, Milpitas, Calif.).

Rats with a fasting blood glucose level on day 5 of less than $350 \mathrm{mg} / \mathrm{dl}$ received a fourth dose of streptozotocin. This procedure was repeated every other day until the target blood glucose level was achieved. Rats in the nondiabetic group received three vehicle injections. ${ }^{1,11}$ Blood glucose was measured over the duration of the F1 experiment (Fig. 1). Rats were given food and water ad libitum. Body weight and water intake were assessed.

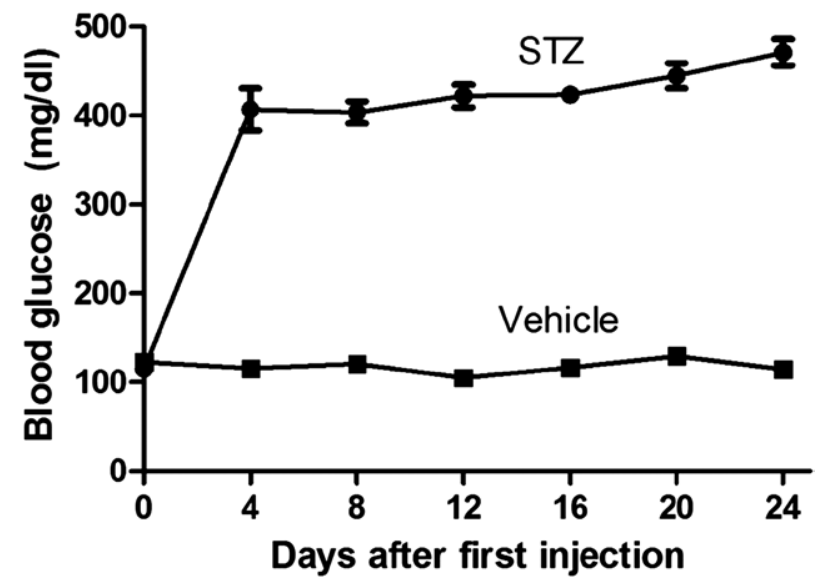

Fig. 1. Streptozotocin dose response curve: average blood glucose (in milligrams per deciliter) for both the streptozotocin ( $n=16$, every 2 days) and vehicle ( $n=8$, every 4 days) rats. There was a significant increase in the mean blood glucose level for the streptozotocin group compared with the vehicle group for all days except day 0 ( $\left.{ }^{*} p<0.0001\right)$. STZ, streptozotocin.

\section{Femur Implant and Inoculation}

Fourteen days after becoming diabetic, rats were anesthetized with $2 \%$ isoflurane in oxygen. The right hind limb skin was shaved, prepared with chlorhexidine and $70 \%$ ethanol, and draped. A 2.5-cm incision was made on the lateral aspect of the right thigh parallel to the femur between the hip and the knee. The plane between the flexor and extensor musculature was sharply and bluntly dissected. The anterolateral aspect of the femur was exposed and a pilot hole was drilled through the proximal cortex of the femoral diaphysis using a $1.5-\mathrm{mm}$ diameter bit. The sterilized fracture plate was screwed onto the femur. The right femur and implant were inoculated by pipetting $10 \mu \mathrm{l}$ of $S$. aureus at $2 \times 10^{8}$ colony-forming units $/ \mathrm{ml}$ onto the fracture plate. The wound was then closed in two layers, with the fascia sutured with running 4-0 Maxon (Covidien, Mansfield, Mass.) and then the skin sutured with interrupted polypropylene. The procedure was repeated for the left hind limb without bacterial inoculation. All rats received a subcutaneous dose of $2.5 \mathrm{mg} / \mathrm{kg}$ flunixin at the time of surgery and then daily for 3 days.

\section{Explantation}

Seven days after implantation, rats were anesthetized and the skin was shaved and prepared as above. The left hind limb sutures were removed and a new incision was made directly over the previous wound. The femur was then exposed using blunt dissection. The vastus lateralis muscle 
Volume 134, Number 3 • Diabetic Rat Fracture Plate Infection

directly overlying the implant was excised in line with the ends of the plate roughly approximating the size and shape of the fracture plate. The muscle was then divided, with the proximal segment used to calculate a wet-to-dry weight and the distal segment used for bacterial quantification. The screw and plate were removed and placed in a sterile container for biofilm quantification. This procedure was repeated for the right side.

\section{Explant Quantitative Microbiology}

The proximal muscle was weighed wet and then dried at $50^{\circ} \mathrm{C}$ for more than 24 hours. The specimen taken for culture was weighed wet, minced with dissection scissors, and homogenized by adding a volume of sterile phosphate-buffered saline equal to 10 times the specimen weight $(10 \times$ dilution, volume to weight). The homogenate underwent six serial 10-fold dilutions, and $100 \mu \mathrm{l}$ of each dilution was plated onto tryptic soy agar plates and incubated for 72 hours at $37^{\circ} \mathrm{C}$. After 72 hours, the plates containing between 30 and 300 colonies were counted for number of colony-forming units.

\section{Biofilm Assay}

Biofilm formation on the explanted hardware was quantified using the technique of Christensen et al. ${ }^{16}$ and modified by Antoci et al. ${ }^{17}$ Explanted stainless steel implants and cortical screws were transferred into a sterile Falcon 24-well plate (Becton, Dickinson and Company, Franklin Lakes, N.J.) and washed six times with sterile phosphatebuffered saline. The hardware was transferred into a glass Vacutainer (Becton, Dickinson) containing $1 \mathrm{ml}$ of tryptic soy broth plus $1 \%$ glucose and sonicated for 5 minutes at $37^{\circ} \mathrm{C}$. Three $300-\mu \mathrm{l}$ aliquots of the sonicated broth were transferred into separate wells on the Falcon plate and incubated overnight at $37^{\circ} \mathrm{C}$. Broth was aspirated and each well was washed three times with sterile phosphatebuffered saline. Crystal violet ( $300 \mu \mathrm{l}$ of $1 \%$ weight to volume in phosphate-buffered saline) was added and incubated for 5 minutes at room temperature. The stain was aspirated and wells were washed three times with sterile phosphate-buffered saline. Finally, $300 \mu \mathrm{l}$ of glacial acetic acid was added to the wells to resuspend the crystal violet stain. Absorbance was measured at $595 \mathrm{~nm}$ using a Genios spectrophotometer running Magellan software (Tecan, Männedorf, Switzerland).

\section{Study Design}

Twenty-four rats were divided into two experimental groups, diabetic $(n=16)$ and nondiabetic $(n=8)$. All animals received bilateral femur implants, and the right hind limb of each rat was inoculated with $S$. aureus, resulting in four rat hind limb groups. The nondiabetic noninoculated data served to quantify infectious burden without direct inoculation in nondiabetic rats. The diabetic noninoculated data were compared to the nondiabetic noninoculated data to determine whether having diabetes alone in the absence of direct inoculation increases infectious susceptibility. The nondiabetic inoculated data served as a control to see whether bacterial inoculation produces measurable infection in a nondiabetic animal. The diabetic inoculated data were compared to the nondiabetic inoculated group to see whether having diabetes has an effect on the immune system's ability to fight and clear infection. In addition, we implanted hardware bilaterally into control rats without bacterial inoculation. These data were compared to the nondiabetic noninoculated data to see whether measurable crossover of bacteria occurs between the inoculated and noninoculated hind limbs.

\section{Statistical Analysis}

The median, 25th quartile, and 75th quartile colony-forming units per milligram of tissue values for the diabetic and nondiabetic rats were calculated using JMP software (SAS Institute, Inc., Cary, N.C.). Because the microbiologic and biofilm data were not distributed normally, they were compared using a Wilcoxon rank sum test. Blood glucose and body weight data were compared using the $t$ test at day 0 and every fourth day.

\section{RESULTS}

\section{Blood Glucose}

Fasting blood glucose measurements for the streptozotocin (diabetic) and vehicle (nondiabetic) groups were averaged for each day (Fig. 1). We observed a significant difference in the mean blood glucose levels between both groups for all days except day $0(p<0.001)$. Average blood glucose for the diabetic group 14 days past the initial hyperglycemic measurement was $466 \pm 16 \mathrm{mg} / \mathrm{dl}$ (mean \pm SEM). The nondiabetic group remained normoglycemic at $105 \pm 11 \mathrm{mg} /$ dl 12 days after receiving the initial vehicle injection (Fig. 1).

\section{Body Weight}

Body weight was averaged by day and by group (Fig. 2). We observed a significant difference in the mean body weight for all days except day 0 F2 
Plastic and Reconstructive Surgery • October 2014

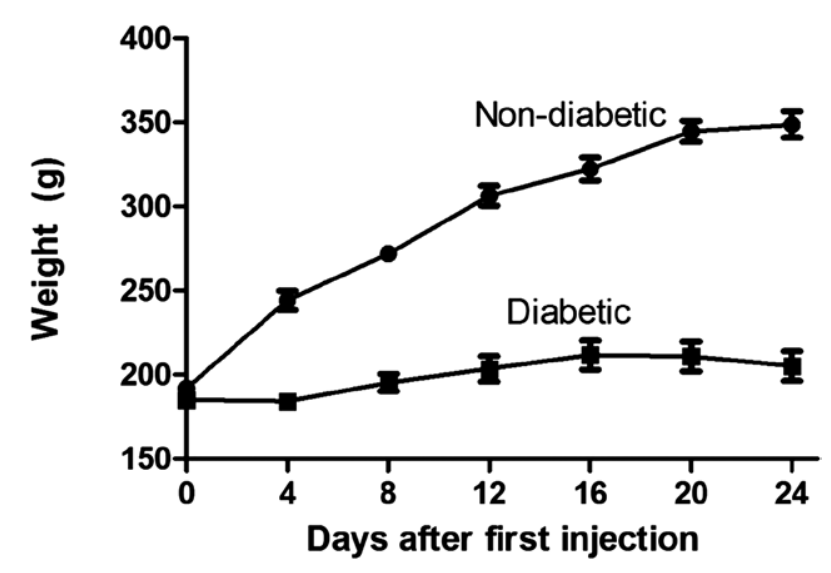

Fig. 2. Average body weight (in grams) for both the diabetic ( $n=16$, every 2 days) and nondiabetic ( $n=8$, every 4 days) rats. There was a significant difference in the mean body weight between the diabetic and nondiabetic groups for all days except day $0(p<0.0001)$.

$(p<0.001)$. Average body weight on day 24 was $349 \pm 8 \mathrm{~g}$ for the nondiabetic group and $205 \pm 9 \mathrm{~g}$ for the diabetic group (Fig. 2).

\section{Quantitative Microbiology}

Infectious burden was measured by quantifying colony-forming units of $S$. aureus 72 hours after muscle explantation (Figs. 3 and 4). We observed a significantly greater infectious burden (colonyforming units per milligram of dry tissue) in the inoculated hind limb for the diabetic group compared with the nondiabetic group $(p=0.0003)$ (Fig. 3). The median number of colony-forming units per milligram of dry tissue in the diabetic group was approximately six orders of magnitude greater than in the nondiabetic group (Table 1). The median colony-forming units per milligram of dry tissue for the noninoculated limb in the diabetic rats was consistently above that of the nondiabetic rats; however, this difference was not significant (Fig. 3 and Table 1). As expected, when we compared the bacterial burden for the inoculated versus noninoculated hind limbs within each group, we detected a significant increase in the colony-forming units per milligram of dry tissue for the inoculated limb (Table 1). A significant difference in the colony-forming units per milligram of dry tissue for the noninoculated hind limb in the diabetic inoculated group compared with the control (diabetic noninoculated) group $(p=0.64)$ (Fig. 4) was not observed, indicating lack of bacterial crossover.

Of the 24 rats in this study, one diabetic rat had to be killed prematurely following unintended femur fracture, and one nondiabetic rat died prematurely of an unknown cause. In addition, the
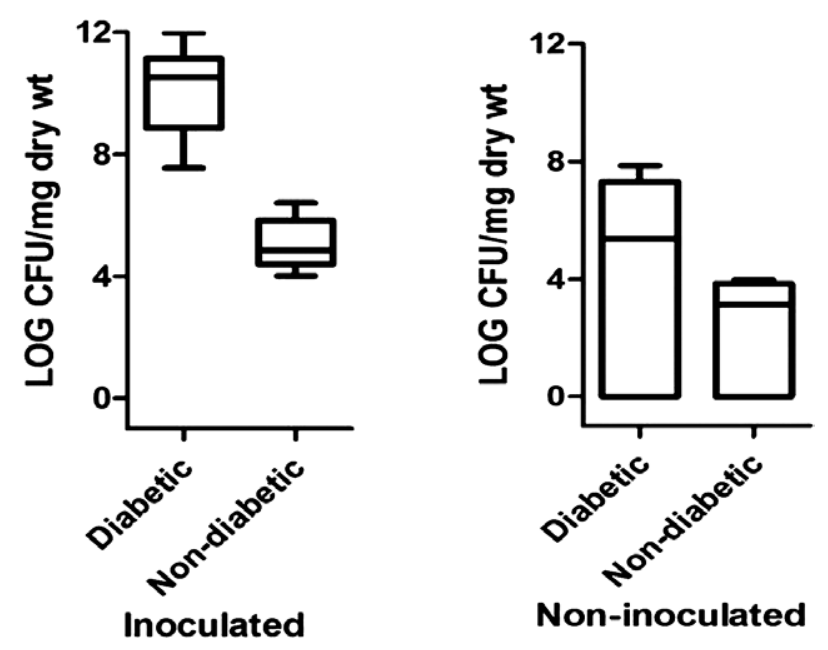

Fig. 3. Infectious burden in the inoculated (left) and noninoculated hind limb (right) for the diabetic $(n=14)$ and nondiabetic $(n=7)$ groups quantified by plate culture method. Box plot data represent median, 25th percentile, and 75th percentile, with whiskers representing minimum and maximum values. Vertical axis is logarithmic base 10 scale, with values representing power of the base. There was a significant difference in the concentration of bacteria cultured for the inoculated hind limb between the diabetic and nondiabetic groups ( $p=0.0003)$. There was not a significant difference detected in the infectious burden for the noninoculated hind limb between the diabetic and nondiabetic groups ( $p=0.0682)$.

culture plates of one rat in the diabetic group became contaminated, and those plates were not used for data analysis. Consequently, data from 14 diabetic and seven nondiabetic animals were analyzed.

\section{Biofilm Formation}

We observed a significant difference in the normalized absorbance between the diabetic (1.37; interquartile range, 1.11 to 2.06 ) and nondiabetic (0.92; interquartile range, 0.88 to 0.94 ; $p=0.0017$ ) (Fig. 4) groups. Data are presented as median (25th to 75 th percentile). Differences in variability in the diabetic and nondiabetic groups can most likely be attributed to the severity of the disease state in diabetic animals, in particular, variable levels of hyperglycemia, which influences bacterial adherence and biofilm formation.

\section{DISCUSSION}

\section{Streptozotocin and Diabetes}

Streptozotocin causes selective pancreatic beta cell toxicity by means of GLUT-2 receptor-associated uptake. ${ }^{18}$ Single large doses produce diabetes by direct cellular toxicity, whereas multiple lower 
Volume 134, Number 3 • Diabetic Rat Fracture Plate Infection

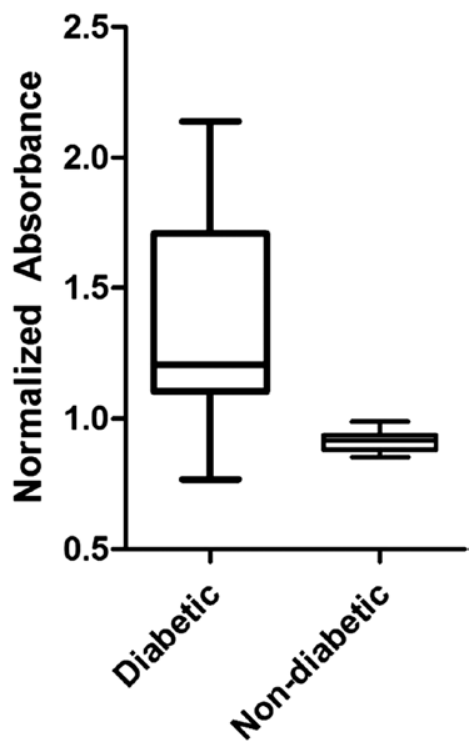

Fig. 4. Normalized absorbance of hardware as measured by indirect staining of biofilm adherent to the fracture plate and screws in both the diabetic and the nondiabetic groups. Box plot data represent median, 25th percentile, and 75th percentile, with whiskers representing \pm 1.5 times the interquartile range. Data represent values for the hardware of the inoculated hind limb of each group after normalization to the hardware of the noninoculated hind limb. Dashed red line has a value of 1 and represents the noninoculated hind limb absorbance data. There was a significant increase in the amount of biofilm detected in the inoculated hind limb of the diabetic rats $(p=0.0017)$.

Table 1. Infectious Burden*

\begin{tabular}{lccc}
\hline & Diabetic & Nondiabetic & $\boldsymbol{p}$ \\
\hline Inoculated & $3.4 \times 10^{10}$ & $7.2 \times 10^{4}$ & $0.0003^{*}$ \\
Noninoculated & $2.6 \times 10^{5}$ & $1.3 \times 10^{3}$ & 0.0682 \\
$p$ & $<0.0001^{*}$ & $0.0017^{*}$ &
\end{tabular}

*Infectious burden in the diabetic and nondiabetic groups for the inoculated and noninoculated hind limbs (data represent median colony-forming units per milligram of dry tissue). We detected a significant increase in the infectious burden in the diabetic group for the inoculated hind limb when compared with the nondiabetic group $(p=0.003)$. No significant difference in the infectious burden between groups for the noninoculated side was detected $(p=0.0682)$. We detected a significant difference in infectious burden between the inoculated and noninoculated sides for both diabetic and nondiabetic groups $(p<0.0001$ and $p=0.0017$, respectively).

doses produce diabetes by means of secondary autoimmune insulitis, better mimicking human type 1 diabetes. Importantly, at low doses (40 mg/ $\mathrm{kg}$ ), streptozotocin is not broadly cytotoxic and is not known to directly cause immune dysfunction.

We induced diabetes in 100 percent of the rats, exceeding results in the literature of 50 to 90 percent for single-dose or multiple low-dose regimens. ${ }^{19}$ The rats tolerated the intraperitoneal injections well, with none exhibiting direct drug toxicity. No rats exceeded $600 \mathrm{mg} / \mathrm{dl}$ or required exogenous insulin.

With the variety of diabetic models available, choosing an experimental model requires careful consideration. Generally, drug-induced type 1 diabetes models are the most reproducible and efficient means of generating a stable diabetes..$^{20,21}$ Type 1 diabetes can also be selected for through inbreeding (i.e., with nonobese diabetic mice). ${ }^{22}$ However, spontaneous models are fairly heterogeneous and contain numerous genes related to autoimmune susceptibility. ${ }^{23,24}$ Type 2 diabetes is defined by insulin resistance with or without impaired insulin secretion caused by obesity and genetic and environmental factors. ${ }^{22-24}$ Because of the complexity of human type 2 diabetes, no single animal model is currently capable of representing all aspects of the disease. The specific genotype and phenotype selected for the disease may differ greatly and confound experimental results. Thus, a type 1 diabetic model seemed the most suitable for this investigation. The choice of chemical induction with streptozotocin is attributable to its efficacy, reproducibility, and controllability. ${ }^{22}$

Our type 1 diabetic model is a very helpful tool for "diabetic" interrogations. However, type 2 diabetes mellitus represents the greater portion of the diabetic population. Therefore, the development of a reliable type 2 model needs to be goal of future diabetes research.

\section{Body Weight}

Trends in body weight were monitored to ensure that no animals experienced wasting secondary to severe hyperglycemia. The weight gain in the control group was significantly greater in comparison with the modest gain seen in the diabetic group. We attribute this small gain to the stresses of the disease state.

\section{Bacterial Quantification}

We consistently reproduced our primary endpoint of quantifying $S$. aureus infections at the surgical site 7 days after plate implantation and bacterial inoculation. Showing a decreased ability to fight postoperative infections, our data align with the abundant literature on diabetic pathophysiology in rats. Secondary manifestations of diabetes such as immune dysfunction and impaired wound healing explain the increased infectious burden seen in our study.

With respect to immune dysfunction, diabetes can best be thought of as a mild immunocompromised state, with most immunologic defects 
Plastic and Reconstructive Surgery • October 2014

occurring in the cellular innate immune system. ${ }^{19}$ Multiple dysfunctions in both neutrophils and macrophages exist (Table 2). Neutrophil dysfunction includes altered activation, increased cell adhesion, altered chemotaxis, and decreased phagocytosis. ${ }^{20}$ Macrophage dysfunction includes decreased cell count, immunogenicity, phagocytic rate, and antigen-presenting ability. ${ }^{21}$ With respect to wound healing, our group recently published a review addressing the impaired deep tissue healing at the site of implantation in diabetic animals. ${ }^{25}$ To date, over 100 known pathophysiologic factors have been discovered that contribute to impaired wound healing in individuals with diabetes. ${ }^{26}$ These include but are not limited to decreased or impaired growth factor secretion, ${ }^{27-29}$ angiogenic response, ${ }^{29,30}$ collagen accumulation, quality of granulation tissue,${ }^{29}$ and delayed bone healing. ${ }^{31-36}$ Delayed bone healing can lead to malunion and subsequently hardware fracture requiring revision surgery. Decreased blood flow to the site of implantation predisposes to infection. Immunologic dysfunction and delayed wound healing contribute to the increased postoperative infectious burden of the diabetic patient. Importantly, the rodent immunologic dysfunction observed here must be interpreted cautiously because the immune response to infection seen in humans could differ. ${ }^{37,38}$

Although our surgical model is novel, we are not the first group to look at in vivo infection susceptibility to $S$. aureus in diabetic rodents. S. aureus injection in the hind paw of both type 1 and type 2 diabetic rodents causes a significantly higher mean bacterial burden compared with nondiabetics. ${ }^{39}$ Importantly, this study also showed that strict glucose regulation improved immune function in the diabetic animals. Although the degree of immune dysfunction seems proportional to blood glucose, further research is necessary to investigate whether control of blood glucose levels with exogenous insulin affects the infectious burden in our model.

\section{Biofilm Formation}

Biofilm formation is of interest to our study because its presence makes treating orthopedic implant infections extremely difficult. Because of the ability of $S$. aureus to generate biofilm on hardware, we indirectly quantified the degree of biofilm formation on our implants. Our results showed a significant increase in the ability of $S$. aureus to form a biofilm on the implanted hardware in the diabetic animals. It is known that the majority of biofilm formation occurs during the early postoperative period. Thus, immediately after bacterial inoculation is the critical period when the body must prevent bacterial adherence to the implanted hardware. However, the early immune response to infection is dominated by neutrophils, and without an intact innate cellular immune response, S. aureus is able to freely colonize the implant. Importantly, normalizing the absorbance values was required because of intraexperimental variability in the absolute absorbance values detected by our spectrophotometer. We believe the relative values obtained are reliable because the inoculated and noninoculated sides for a given animal were analyzed simultaneously.

\section{Study Limitations}

In our study, we are limited in drawing conclusions about the infectious susceptibility of our diabetic animals compared with nondiabetics. Insight into infectious susceptibility comes from the bacterial quantification data in the noninoculated hind limb. One would assume that the noninoculated hind limbs of both groups would have approximately the same degree of bacterial burden if their infectious susceptibility was the same. However, this was not the case, as the diabetic group had increased bacterial burden in the noninoculated hind limb compared with the nondiabetic group. Even in the absence of direct inoculation, the diabetic group was more susceptible to a more severe postoperative infection than the nondiabetic group. This trend is consistent

Table 2. Immune Dysfunctions*

\begin{tabular}{lcc}
\hline & Humoral & \multicolumn{1}{c}{ Cellular } \\
\hline Innate & Complement & Neutrophils (PMN) \\
& Cytokines without stimulation & Macrophages/monocytes \\
Adaptive & Antibodies & $\downarrow$ \\
Adherence & $\uparrow$ & T lymphocytes \\
*Summary of the immune dysfunctions found in diabetic patients. [Adapted from Geerlings SE, Hoepelman AI. Immune dysfunction in \\
patients with diabetes mellitus (DM). FEMS Immunol Med Microbiol. 1999;26:259-265.] Up and down arrows represent increased or decreased \\
function and/or quantity. An equal sign represents no significant difference between diabetics and nondiabetics. \\
PMN, polymorphonuclear neutrophil.
\end{tabular}




\section{Volume 134, Number 3 • Diabetic Rat Fracture Plate Infection}

with recent reports in the literature that point to a variety of factors that contribute to infection susceptibility, ${ }^{14,25}$ including decreased inflammatory response, poor angiogenic response, and nitric oxide deficiency. Insufficient power is likely why the two groups could not be distinguished, as the sample size for our study was small. In addition, the duration of the study may affect the bioburden, as it is possible that the infection for the diabetic animals would worsen over time and the infection in control subjects may clear. In contrast, the difference between the bioburden of the inoculated and noninoculated nondiabetic rats was statistically significant. This discrepancy may be attributed to the variability of the infectious burden in the diabetic noninoculated group. Although some diabetic wounds exhibited considerable infection in the absence of inoculation, others had none. In contrast, data obtained from nondiabetic rats were much less variable. The severity of the disease state in the diabetic rats may thus influence the variability. Rats with more severe hyperglycemia $(>500 \mathrm{mg} / \mathrm{dl})$ may exhibit greater immune deficiency, resulting in increased infectious burden in comparison with rats with moderate hyperglycemia (350 to $500 \mathrm{mg} / \mathrm{dl}$ ). Importantly, we attribute the bacterial growth in the noninoculated hind limb of both groups to normal bacterial burden from surgery, even though every effort was made to ensure sterility for the entirety of the operations.

Our study was limited in its ability to quantify total infectious burden at the surgical site, as we harvested only the muscle directly overlying the fracture plate. Although it is likely that the infections were not localized, the technique we used provided the accuracy and precision needed to detect statistically significant differences by means of the tissue plate quantification method. Lastly, our model of directly inoculating the surgical site with concentrated bacteria does not mimic reallife acquisition of a surgical infection.

\section{CONCLUSIONS}

The extensive literature on immune dysfunction and delayed wound healing in diabetic patients emphasizes the need to study postoperative infections in diabetic animals. The novel outcomes-based streptozotocin dosing regimen presented here induced diabetes in 100 percent of the rats and achieved consistent hyperglycemic values. Diabetic rats demonstrated elevated infection proclivity above nondiabetics after $S$. aureus inoculation in the presence of implanted hardware. These data support the hypothesis that uncontrolled type 1 diabetes adversely affects the immune system's ability to fight and clear bacteria in the postoperative period.

Bruce Klitzman, Ph.D.

Box 3906

Duke University Medical Center Durham, N.C. 27710

klitz@duke.edu

\section{ACKNOWLEDGMENTS}

This work was funded in part by National Institutes of Health grants EB000708 and T32 GM08555 and by the Robert Jones Fund. The authors thank Dr. Howie Levinson, Dr. Scott Nichols, Julia Skettini, and Reema Sil, whose help and suggestions were greatly appreciated.

\section{REFERENCES}

1. U.S. Department of Health and Human Services. National diabetes fact sheet: general information and national estimates on diabetes in the United States. Available at: http:// www.cdc.gov/diabetes/pubs/factsheet07.htm. Accessed September 27, 2010.

2. Botushanov NP, Orbetzova MM. Bone mineral density and fracture risk in patients with type 1 and type 2 diabetes mellitus. Folia Med (Plovdiv) 2009;51:12-17.

3. Khazai NB, Beck GR Jr, Umpierrez GE. Diabetes and fractures: An overshadowed association. Curr Opin Endocrinol Diabetes Obes. 2009;16:435-445.

4. Schwartz AV. Diabetes mellitus: Does it affect bone? Calcif Tissue Int. 2003;73:515-519.

5. Darouiche RO. Treatment of infections associated with surgical implants. N Engl J Med. 2004;350:1422-1429.

6. Arciola CR, An YH, Campoccia D, Donati ME, Montanaro L. Etiology of implant orthopedic infections: A survey on 1027 clinical isolates. In J Artif Organs 2005;28:1091-1100.

7. Moon HK, Han CD, Yang IH, Cha BS. Factors affecting outcome after total knee arthroplasty in patients with diabetes mellitus. Yonsei Med J. 2008;49:129-137.

8. Whitehouse JD, Friedman ND, Kirkland KB, Richardson WJ, Sexton DJ. The impact of surgical-site infections following orthopedic surgery at a community hospital and a university hospital: Adverse quality of life, excess length of stay, and extra cost. Infect Control Hosp Epidemiol. 2002;23:183-189.

9. Weinstein RA, Darouiche RO. Device-associated infections: A macroproblem that starts with microadherence. Clin Infect Dis. 2001;33:1567-1572.

10. Zalavras CG, Christensen T, Rigopoulos N, Holtom P, Patzakis MJ. Infection following operative treatment of ankle fractures. Clin Orthop Relat Res. 2009;467:1715-1720.

11. SooHoo NF, Krenek L, Eagan MJ, Gurbani B, Ko CY, Zingmond DS. Complication rates following open reduction and internal fixation of ankle fractures.J Bone Joint Surg Am. 2009;91:1042-1049.

12. SooHoo NF, Farng E, Lieberman JR, Chambers L, Zingmond DS. Factors that predict short-term complication rates after total hip arthroplasty. Clin Orthop Relat Res. 2010;468:2363-2371.

13. Fang A, Hu SS, Endres N, Bradford DS. Risk factors for infection after spinal surgery. Spine (Phila Pa 1976) 2005;30:1460-1465. 


\section{Plastic and Reconstructive Surgery • October 2014}

14. Chen S, Anderson MV, Cheng WK, Wongworawat MD. Diabetes associated with increased surgical site infections in spinal arthrodesis. Clin Orthop Relat Res. 2009;467:1670-1673.

15. Marchant MH Jr, Viens NA, Cook C, Vail TP, Bolognesi MP. The impact of glycemic control and diabetes mellitus on perioperative outcomes after total joint arthroplasty. $J$ Bone Joint Surg Am. 2009;91:1621-1629.

16. Christensen GD, Simpson WA, Younger JJ, et al . Adherence of coagulase-negative staphylococci to plastic tissue culture plates: A quantitative model for adherence of staphylococci to medical devices. J Clin Microbiol. 1985;22:996-1006.

17. Antoci V Jr, Adams CS, Parvizi J, et al. The inhibition of Staphylococcus epidermidis biofilm formation by vancomycinmodified titanium alloy and implications for the treatment of periprosthetic infection. Biomaterials 2008;29:4684-4690.

18. Tesch GH, Allen TJ. Rodent models of streptozotocin-induced diabetic nephropathy. Nephrology (Carlton) 2007;12:261-266.

19. Geerlings SE, Hoepelman AI. Immune dysfunction in patients with diabetes mellitus (DM). FEMS Immunol Med Microbiol. 1999;26:259-265.

20. Alba-Loureiro TC, Munhoz CD, Martins JO, et al. Neutrophil function and metabolism in individuals with diabetes mellitus. Braz J Med Biol Res. 2007;40:1037-1044.

21. Ma H, Liu G, Ding W, Wu Y, Cai L, Zhao Y. Diabetes-induced alteration of F4/80+ macrophages: A study in mice with streptozotocin-induced diabetes for a long term. $J$ Mol Med (Berl.) 2008;86:391-400.

22. Rees DA, Alcolado JC. Animal models of diabetes mellitus. Diabet Med. 2005;22:359-370.

23. von Herrath M, Nepom GT. Animal models of human type 1 diabetes. Nat Immunol. 2009;10:129-132.

24. Junod A, Lambert AE, Stauffacher W, Renold AE. Diabetogenic action of streptozotocin: Relationship of dose to metabolic response. J Clin Invest. 1969;48:2129-2139.

25. Le NN, Rose MB, Levinson H, Klitzman B. Implant healing in experimental animal models of diabetes. JDiabetes Sci Technol. 2011;5:605-606.

26. Brem H, Tomic-Canic M. Cellular and molecular basis of wound healing in diabetes. JClin Invest. 2007;117:1219-1222.

27. Galkowska H, Wojewodzka U, Olszewski WL. Chemokines, cytokines, and growth factors in keratinocytes and dermal epithelial cells in the margin of chronic diabetic food ulcers. Wound Repair Regen. 2006;14:558-565.

28. Goren I, Müller E, Pfeilschifter J, Frank S. Severely impaired insulin signaling in chronic wounds of diabetic ob/ob mice: A potential role of tumor necrosis factor-alpha. Am J Pathol. 2006;168:765-777.

29. Falanga V. Wound healing and its impairment in the diabetic foot. Lancet 2005;366:1736-1743.

30. Galiano RD, Tepper OM, Pelo CR, et al. Topical vascular endothelial growth factor accelerates diabetic wound healing through increased angiogenesis and by mobilizing and recruiting bone marrow-derived cells. Am J Pathol. 2004;164:1935-1947.

31. Loder RT. The influence of diabetes mellitus on the healing of closed fractures. Clin Orthop Relat Res. 1988;232:210-216.

32. Follak N, Klöting I, Merk H. Influence of diabetic metabolic state on fracture healing in spontaneously diabetic rats. Diabetes Metab Res Rev. 2005;21:288-296.

33. Botolin S, Faugere MC, Malluche H, Orth M, Meyer R, McCabe LR. Increased bone adiposity and peroxisomal proliferator-activated receptor-gamma2 expression in type I diabetic mice. Endocrinology 2005;146:3622-3631.

34. Kasahara T, Imai S, Kojima H, et al. Malfunction of bone marrow-derived osteoclasts and the delay of bone fracture healing in diabetic mice. Bone 2010;47:617-625.

35. Azad V, Breitbart E, Al-Zube L, Yeh S, O'Connor JP, Lin SS. rhBMP-2 enhances the bone healing response in a diabetic rat segmental defect model. J Orthop Trauma 2009;23:267-276.

36. Ogasawara A, Nakajima A, Nakajima F, Goto K, Yamazaki M. Molecular basis for affected cartilage formation and bone union in fracture healing of the streptozotocin-induced diabetic rat. Bone 2008;43:832-839.

37. Roep BO. Are insights gained from NOD mice sufficient to guide clinical translation? Another inconvenient truth. Ann N Y Acad Sci. 2007;1103:1-10.

38. Mestas J, Hughes CC. Of mice and not men: Differences between mouse and human immunology. I Immunol. 2004;172:2731-2738.

39. Park S, Rich J, Hanses F, Lee JC. Defects in innate immunity predispose C57BL/6J-Leprdb/Leprdb mice to infection by Staphylococcus aureus. Infect Immun. 2009;77:1008-1014. 


\section{AUTHOR QUERIES}

\section{AUTHOR PLEASE ANSWER ALL QUERIES}

AQ1-References 6-9 and 19-24 were renumbered according to order of citation in text, per Journal style. Please confirm that edits are correct.

AQ2-TSP spelled correctly here and throughout as "tryptic soy broth"? If not, please revise as needed.

AQ3-Names and city/state locations of manufacturers correct, here and throughout? If not, please supply the correct information, per Journal style for use of brand and manufacturer names.

AQ4-No dashed red line is evident in Figure 4. Please revise figure legend as needed.

AQ5-The footnote to Table 2 indicates that this table has been adapted. Please provide a copy of the permission letter from the publisher of FEMS Immunol Med Microbiol granting permission to adapt the original table. Please note that we cannot publish this adapted table without permission.

AQ6-Sentence that begins "Insufficient power": Correct as edited? Please advise/revise as needed. 